\title{
Effect of Cold Working Ratio on Function Deterioration Process of Ti-Ni Shape Memory Alloy
}

\author{
H. Cho, M. Yamaguchi, T. Yamamoto and T. Sakuma \\ Faculty of Engineering, Oita University, 700 Dannoharu,Oita 870-1192, Japan \\ Fax: 81-97-554-7771, e-mail: hirocho@cc.oita-u.ac.jp
}

\begin{abstract}
To investigate the effect of cold working ratio on function deterioration process of Ti-Ni shape memory alloy, repeated loading-unloading tests with constant strain condition were carried out. The chemical composition of specimen is Ti-50.3at $\% \mathrm{Ni}$ and cold working ratio $(\mathrm{CW})$ is $30.1 \%$ $43.0 \%$ and $51.0 \%$. Heat-treatment condition is at $673 \mathrm{~K}$ for $3.6 \mathrm{ks}$. The decrement of recovery strain decreases with increasing $\mathrm{CW}$. Moreover, lower $\mathrm{CW}$ leads to the decreasing of the number of loading-unloading cycle which decrement of recovery strain slowdown starts. Furthermore, decrement of maximum applied stress decreases with increasing $\mathrm{CW}$. On the other hand, in spite of critical stress for inducing martensite increases with increasing $\mathrm{CW}$, the decrement of critical stress for inducing martensite is almost same regardless of CW. From these results, increasing of $\mathrm{CW}$ leads increasing of deterioration resistance. These tendencies are caused by the variation of dislocation density and grain size due to the variation of $\mathrm{CW}$.
\end{abstract}

Key words: Shape memory alloy, Ti-Ni alloy, shape memory properties, Function deterioration process

\section{INTRODUCTION}

The Ti-Ni shape memory alloys (SMA) are applied to various fields for example industry and medical, because they have not only superior shape memory properties but also ductility, toughness, fatigue resistance, corrosion resistance and abrasion resistance. Shape memory properties and the super elasticity are repeatedly used in applications of SMA. It is important to investigate the deterioration properties and fatigue properties with the repetition use of SMA. Consequently, many researches about deterioration properties and fatigue properties of the SMA have been performed.

Mechanical properties and shape memory properties of SMA are affected by the stress, strain, temperature, alloy composition and manufacturing processes ${ }^{[6-8]}$. In previous researches, it is reported that the increasing of cold working ratio $(\mathrm{CW})$ leads increasing of critical stress for slip. And due to this, the mechanical properties and shape memory properties are improved ${ }^{[9-12]}$. However, there are few systematic researches about the effect of the $\mathrm{CW}$ on function deterioration process of SMA.

In this study, the effect of cold working ratio on function deterioration process of Ti-Ni shape memory alloy was investigated by repeated loading-unloading cycle tests with constant strain condition.

\section{EXPERIMANTAL PLOCEDURE}

The chemical composition of the alloy used in this study is Ti-50.3at\% $\%$ i. The specimen shape is a wire with about $0.75 \mathrm{~mm}$ diameter and $60 \mathrm{~mm}$ gage length. The specimen was processed in the following manner; the Ti-Ni-Cu shape memory alloy ingot was made by using a high frequency induction vacuum furnace, and then was hot forged and hot extruded followed by cold drawing and intermediate annealing. Cold working ratio (CW) of the specimen was $30.1 \%, 43.0 \%$ and $51.0 \%$.
The specimens were performed heat treatment at $673 \mathrm{~K}$ for $3.6 \mathrm{ks}$ in air.

Transformation temperatures (martensite start temperature $M_{s}$, martensite finish temperature $M_{f}$, austenite start temperature $A_{s}$ and austenite finish temperature $A_{f}$ ) of each specimen were measured by differential scanning calorimetry (DSC). Tabel.1 shows transformation temperatures of each specimen.

To investigate the effect of cold working ratio on

Table. 1 Transformation Temperatures of Ti-50.3at\%Ni alloys measured by DSC.

\begin{tabular}{ccccc}
\hline $\mathrm{CW}[\%]$ & $A_{f}[\mathrm{~K}]$ & $A_{s}[\mathrm{~K}]$ & $M_{s}[\mathrm{~K}]$ & $M_{f}[\mathrm{~K}]$ \\
\hline 30.1 & 337.6 & 315.8 & 346.7 & 307.2 \\
43.0 & 332.4 & 295.8 & 327.4 & 297.8 \\
51.0 & 343.3 & 310.4 & 333.7 & 304.7 \\
\hline
\end{tabular}

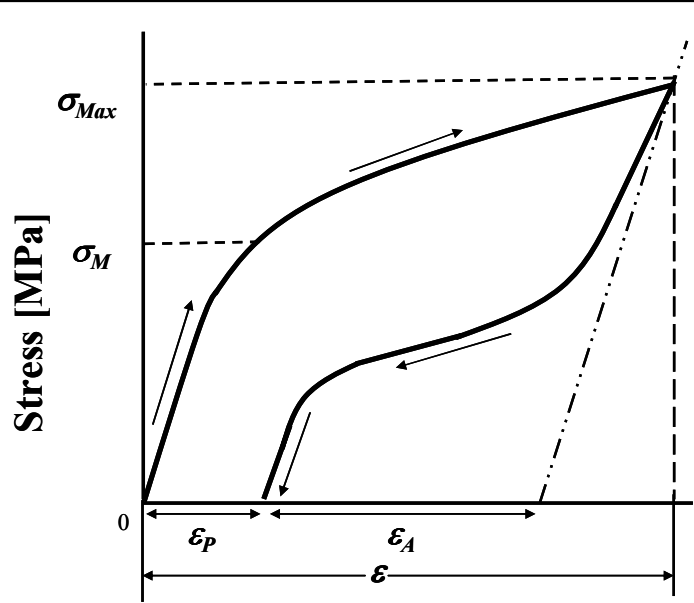

Strain [\%]

Fig. 1 Schematic drawing of stress-strain curve. 
function deterioration process, repeated loading-unloading cycle tests with constant strain condition were carried out. Figure 1 shows the schematic drawing of stress-strain curve in the loading-unloading test with constant strain. The atmosphere temperature is fixed at the temperature which is 30 degrees higher than the $A_{f}$ temperature. The applied strain $(\varepsilon)$ is 3,5 and $8 \%$. The strain rate is $10 \% / \mathrm{sec}$. The number of repeated cycle is $500 . \varepsilon_{A}, \varepsilon p$, $\sigma_{M s}$, and $\sigma_{\max }$ indicate recovery strain, plastic strain, critical stress for induced martensitic phase and maximum loading stress, respectively.

\section{RESULTS AND DISCUSSION}

\subsection{Effect of cold working ratio on recovery strain}

Figure 2 shows relationships between $\varepsilon_{A}(\mathrm{~N}) / \varepsilon_{A}(1)$ and number of cycle for each CW. (a), (b) and (c) show results of $\varepsilon=3 \%, \varepsilon=5 \%$ and $\varepsilon=8 \%$, respectively.

In the case of $\varepsilon=3 \%$ and $\mathrm{CW}=30.1 \%, \varepsilon_{A}(\mathrm{~N}) / \varepsilon_{A}(1)$ decreases with increasing number of cycle, as shown in Fig. 2 (a). When the number of cycle is over 10, the decrement of $\varepsilon_{A}(\mathrm{~N}) / \varepsilon_{A}(1)$ slows down. On the other hand, in the case of $\mathrm{CW}=43.1 \%$ and $51.0 \%, \varepsilon_{A}(\mathrm{~N}) / \varepsilon_{A}(1)$ scarcely decrease at first several cycles. After that, they show the similar tendency in the case of $\mathrm{CW}=30.1 \%$.

However, in the case of $\varepsilon=5 \%$ and $\varepsilon=8 \%, \varepsilon_{A}(\mathrm{~N}) / \varepsilon_{A}(1)$ decreases at the first cycle regardless of $\mathrm{CW}$, as shown in Fig. 2 (b) and (c). Decrement of $\varepsilon_{A}(\mathrm{~N}) / \varepsilon_{A}(1)$ decrease with increasing $\mathrm{CW}$, and the number of cycle at the starting point, which decrement of $\varepsilon_{A}(\mathrm{~N}) / \varepsilon_{A}(1)$ slows down, increases with increasing $\mathrm{CW}$.

From these results, increasing of $\mathrm{CW}$ leads increasing of deterioration resistance. It is thought that the reason of increasing of deterioration resistance is due to the increasing of dislocation density. Increasing of $\mathrm{CW}$ leads to the increasing of dislocation density. Accordingly, introducing dislocation newly becomes hard with increasing $\mathrm{CW}$. Therefore, in the case of the $\mathrm{CW}$ is large $(\mathrm{CW}=43.0 \%$ and $51.0 \%)$, the domain which $\varepsilon_{A}(\mathrm{~N}) / \varepsilon_{A}(1)$ scarcely decreases exists, and decrement of $\varepsilon_{A}(\mathrm{~N}) / \varepsilon_{A}(1)$ decreases. On the other hand, in the case of $\mathrm{CW}$ is small $(\mathrm{CW}=30.1 \%)$, introducing dislocation is newly easier than other specimen. Therefore, decreasing of $\varepsilon_{A}(\mathrm{~N}) / \varepsilon_{A}(1)$ is caused at the first cycle, and the decrement of $\varepsilon_{A}(\mathrm{~N}) / \varepsilon_{A}(1)$ is larger than other specimen. In addition, since introducing dislocation is easily, lower $\mathrm{CW}$ leads to the decreasing of the number of cycle which decrement of $\varepsilon_{A}(\mathrm{~N}) / \varepsilon_{A}(1)$ slowdown starts, and number of cycle to saturate the dislocation density decreases.

\subsection{Effect of cold working ratio on plastic strain}

Figure 3 shows relationships between $\varepsilon_{p}(\mathrm{~N})-\varepsilon_{p}(1)$ and number of cycle of $\varepsilon=3 \%$ for each CW.

In the case of $\varepsilon=3 \%, C W=30.1 \%, \varepsilon_{p}(\mathrm{~N})-\varepsilon_{p}(1)$ increases with increasing the number of cycle, as shown in Fig. 3. When the number of cycle is about

10 , the increment of $\varepsilon_{p}(\mathrm{~N})-\varepsilon_{p}(1)$ slows down. On the other hand, In the case of $\mathrm{CW}=43.1 \%$ and $51.0 \%$, $\varepsilon_{p}(\mathrm{~N})-\varepsilon_{p}(1)$ scarcely increases at first several cycles. After that, they show the similar tendency in the case of $\mathrm{CW}=30.1 \%$. These tendency are accord with the tendency of decreasing of $\varepsilon_{A}(\mathrm{~N}) / \varepsilon_{A}(1)$.
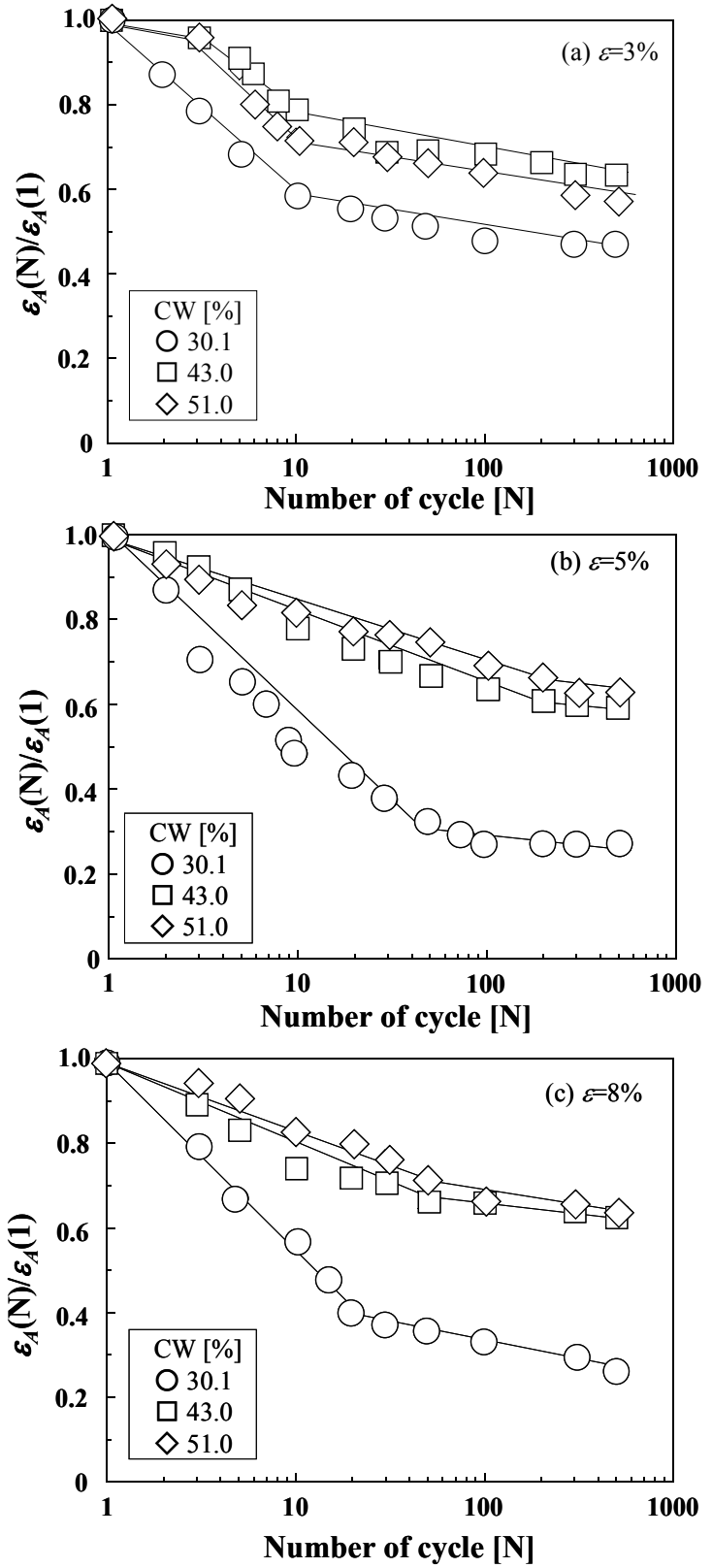

Fig. 2 Relationships between $\varepsilon_{A}(\mathrm{~N}) / \varepsilon_{A}(1)$ and number of cycle for (a) $\varepsilon=3 \%$, (b) $\varepsilon=5 \%$ and (c) $\varepsilon=8 \%$.

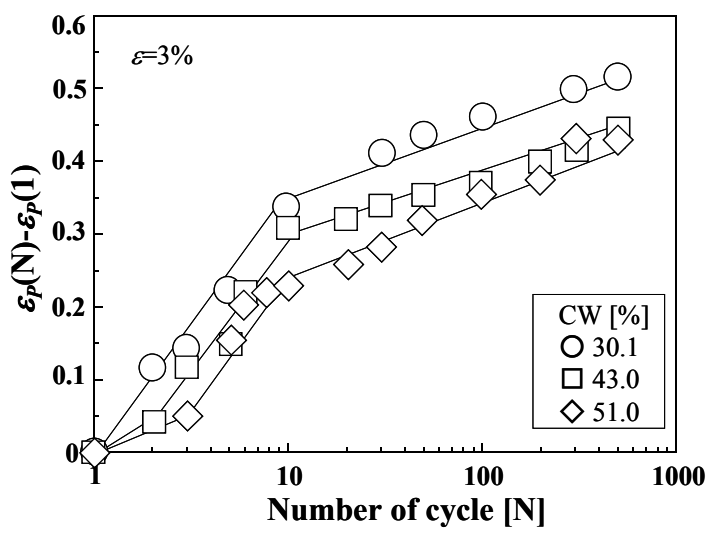

Fig. 3 Relationships between $\varepsilon_{p}(\mathrm{~N})-\varepsilon_{p}(1)$ and number of cycle of $\varepsilon=3 \%$ for each CW. 


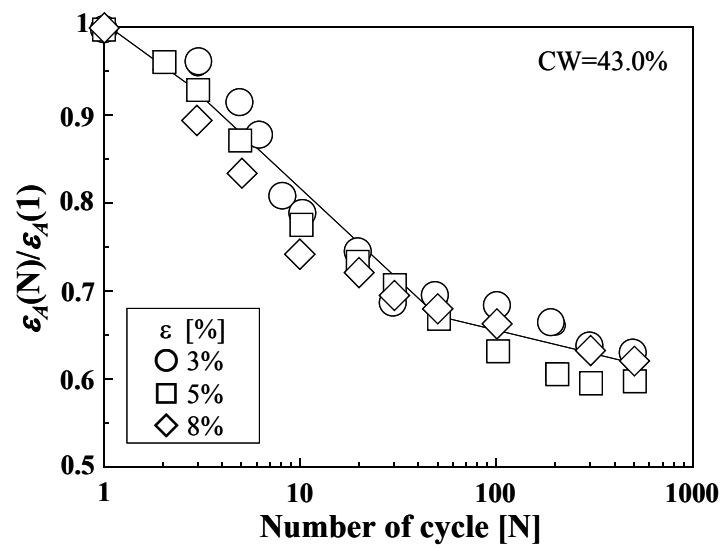

Fig. 4 Relationships between $\varepsilon_{A}(\mathrm{~N}) / \varepsilon_{A}(1)$ and number of cycle of $\mathrm{CW}=43.0 \%$ for each $\varepsilon$.

In the case of $\varepsilon=5 \%$ and $8 \%$, the tendency of increasing of $\varepsilon_{p}(\mathrm{~N})-\varepsilon_{p}(1)$ accords with decreasing of $\varepsilon_{A}(\mathrm{~N}) / \varepsilon_{A}(1)$ similarly as shown in Fig. 2 (b) and (c).

From these results, increasing of $\varepsilon_{p}$ decreases and deterioration resistance increases with increasing $\mathrm{CW}$. Moreover, the tendency of the increasing of $\varepsilon_{p}$ operates together with the tendency of the decreasing of the $\varepsilon_{A}$.

\subsection{Effect of applied strain on recovery strain}

Figure 4 shows relationships between $\varepsilon_{p}(\mathrm{~N})-\varepsilon_{p}(1)$ and number of cycle of $\mathrm{CW}=43.0 \%$ for each $\varepsilon$. The tendency of the decreasing of $\varepsilon_{p}(\mathrm{~N})-\varepsilon_{p}(1)$ is almost same regardless of $\varepsilon$, as shown in Fig.4. In the case of $\mathrm{CW}=30.1 \%$ and $51.0 \%$, decreasing of $\varepsilon_{p}(\mathrm{~N})-\varepsilon_{p}(1)$ is almost same regardless of $\varepsilon$ similarly.

From these result, it is thought that the deterioration resistance is not affected by $\varepsilon$. Thus, the deterioration resistance has a strong influence on $\mathrm{CW}$, and the deterioration resistance has a little influence on $\varepsilon$.

\subsection{Maximum loading stress}

Figure 5shows relationships between $\sigma_{\max }(\mathrm{N}) / \sigma_{\max }(1)$ and number of cycle of $\varepsilon=3 \%$ for each $\mathrm{CW}$. The decrement of $\sigma_{\max }(\mathrm{N}) / \sigma_{\max }(1)$ decrease with increasing $\mathrm{CW}$, as shown in Fig.5. In the case of $\varepsilon=5 \%$ and $8 \%$, the decrement of $\sigma_{\max }(\mathrm{N}) / \sigma_{\max }(1)$ decrease with increasing $\mathrm{CW}$ similarly. It is thought that this tendency caused by increasing of deterioration resistance with

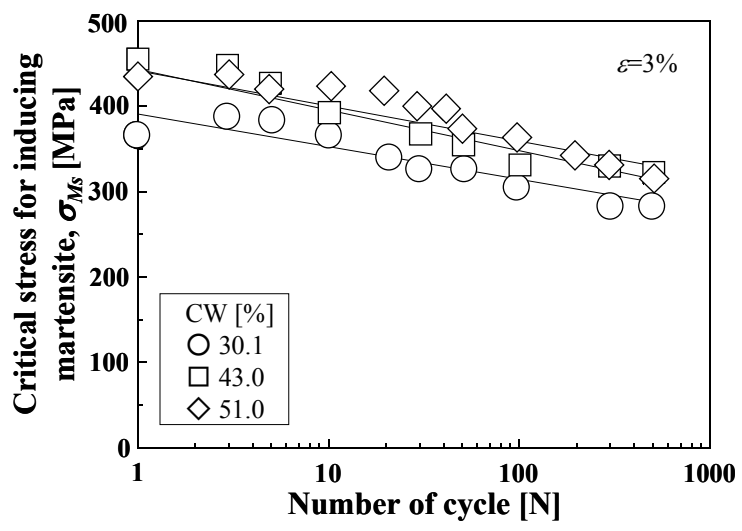

Fig. 6 Relationships between $\sigma_{M_{s}}$ and number of cycle of $\varepsilon=3 \%$ for each $\mathrm{CW}$.

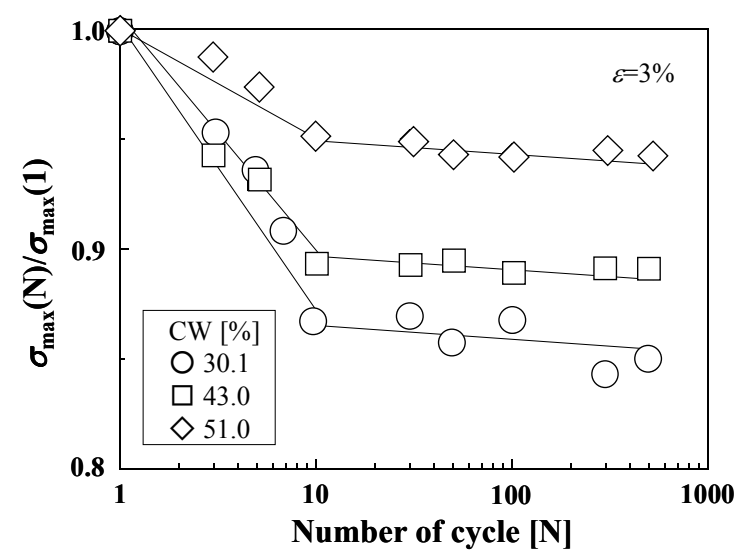

Fig. 5 Relationships between $\sigma_{\max }(\mathrm{N}) / \sigma_{\max }(1)$ and number of cycle of $\varepsilon=3 \%$ for each CW.

increasing dislocation density. Increasing $\mathrm{CW}$ leads to increasing of dislocation density. Increasing of dislocation density leads to reducing of damage by the loading-unloading cycle. Therefore, the decrement of $\sigma_{\max }(\mathrm{N}) / \sigma_{\max }(1)$ decreases with increasing $\mathrm{CW}$.

Furthermore, $\varepsilon_{A}(\mathrm{~N}) / \varepsilon_{A}(1)$ and $\varepsilon_{p}(\mathrm{~N})-\varepsilon_{p}(1)$ do not have a large difference between $\mathrm{CW}=43.0 \%$ and $\mathrm{CW}=51.0 \%$ (as shown in Fig. 2 and Fig. 3). However, $\sigma_{\max }(\mathrm{N}) / \sigma_{\max }(1)$ has a large difference between $\mathrm{CW}=43.0 \%$ and $\mathrm{CW}=51.0 \%$.

From these results, it is thought that the effect of CW (variation of dislocation) on $\varepsilon_{A}(\mathrm{~N}) / \varepsilon_{A}(1)$ and $\varepsilon_{p}(\mathrm{~N})-\varepsilon_{p}(1)$ is saturated when the $\mathrm{CW}$ reach $43.0 \%$. In contrast, the effect of CW on $\sigma_{\max }(\mathrm{N}) / \sigma_{\max }(1)$ is not saturated when the $\mathrm{CW}$ reach $51.0 \%$. Thus the effect of $\mathrm{CW}$ on $\sigma_{\max }(\mathrm{N}) / \sigma_{\max }(1)$ is larger than that of $\varepsilon_{A}(\mathrm{~N}) / \varepsilon_{A}(1)$ and $\varepsilon_{p}(\mathrm{~N})-\varepsilon_{p}(1)$.

\subsection{Critical stress for induced martensitic phase}

Figure 6 shows relationships between $\sigma_{M s}$ and number of cycle of $\varepsilon=3 \%$ for each CW. $\sigma_{M s}$ increases with increasing $\mathrm{CW}$. In the case of $\varepsilon=5 \%$ and $8 \%$, the decrement of $\sigma_{M s}$ increase with increasing CW similarly.

It is thought that this tendency caused by variation of grain size. Increase of $\mathrm{CW}$ leads to decreasing of grain size. The decreasing grain size leads to increasing $\sigma_{M s}$ because of Hall-Petch effect. Increasing number of cycle leads to increasing dislocation density, and so $\sigma_{M s}$

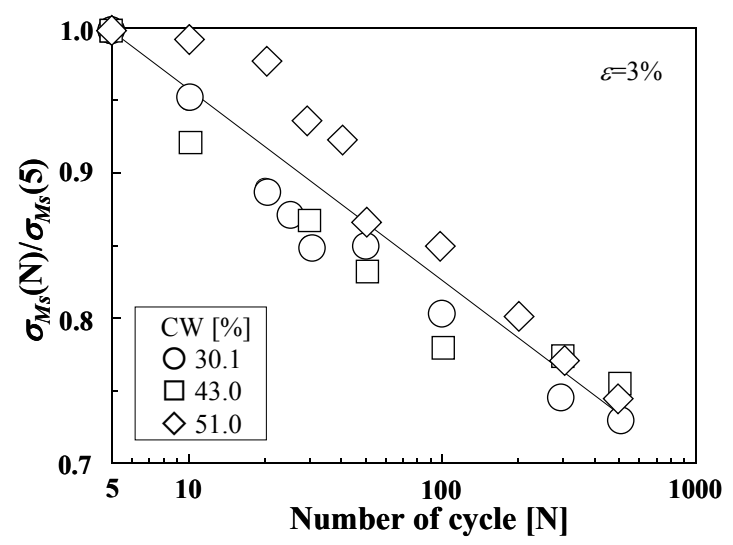

Fig. 7 Relationships between $\sigma_{M s}(\mathrm{~N}) / \sigma_{M s}(1)$ and number of cycle of $\varepsilon=3 \%$ for each $\mathrm{CW}$. 
decreases because the internal stress to lead martensitic transformation is generated by introducing dislocation.

Figure 7 shows relationships between $\sigma_{M s}(\mathrm{~N}) / \sigma_{M s}(5)$ and number of cycle of $\varepsilon=3 \%$ for each CW. $\sigma_{M s}$ was unstable to several cycles of the beginning. Therefore, the $\sigma_{M s}$ was normalized by the results of $\mathrm{N}=5$. The decrement of $\sigma_{M s}(\mathrm{~N}) / \sigma_{M s}(5)$ is almost same regardless of $\mathrm{CW}$, as shown in Fig. 7. In the case of $\varepsilon=5 \%$ and $8 \%$, decrement of $\sigma_{M s}(\mathrm{~N}) / \sigma_{M s}(5)$ is almost same regardless of $\mathrm{CW}$ similarly. From these results, the effect of $\mathrm{CW}$ on decrease of $\sigma_{M s}(\mathrm{~N}) / \sigma_{M s}(5)$ is a little.

\section{CONCLUSION}

The effect of cold working ratio on function deterioration process of $\mathrm{Ti}-50.3 \mathrm{at} \% \mathrm{Ni}$ shape memory alloy was investigated. The obtain results are summarized as follows;

(1) Decrement of $\varepsilon_{A}(\mathrm{~N}) / \varepsilon_{A}(1)$ decreases with increasing $\mathrm{CW}$. Moreover, lower $\mathrm{CW}$ lead to the decreasing of number of cycle which decrement of $\varepsilon_{A}(\mathrm{~N}) / \varepsilon_{A}(1)$ slowdown starts. Thus, increasing of $\mathrm{CW}$ leads increasing of deterioration resistance. Furthermore, decrement of $\varepsilon_{A}(\mathrm{~N}) / \varepsilon_{A}(1)$ is not affected by $\varepsilon$, but $\varepsilon_{A}(\mathrm{~N}) / \varepsilon_{A}(1)$ is affected by $\mathrm{CW}$. These tendencies are caused by the variation of dislocation due to the variation of $\mathrm{CW}$.

(2) The increasing of $\varepsilon_{p}(\mathrm{~N})-\varepsilon_{p}(1)$ accords with decreasing of $\varepsilon_{A}(\mathrm{~N}) / \varepsilon_{A}(1)$. Thus, the $\varepsilon_{p}(\mathrm{~N})-\varepsilon_{p}(1)$ is a reflection of $\varepsilon_{A}(\mathrm{~N}) / \varepsilon_{A}(1)$.

(3) The decrement of $\sigma_{\max }(\mathrm{N}) / \sigma_{\max }(1)$ decrease with increasing $\mathrm{CW}$. On the other hand, in spite of $\sigma_{M s}$ increase with increasing $\mathrm{CW}$, the decrement of $\sigma_{M s}(\mathrm{~N}) / \sigma_{M s}(1)$ is almost same regardless of CW. These tendencies are caused by the variation of dislocation and grain size due to the variation of $\mathrm{CW}$.

\section{REFERENCES}

[1] S. Miyazaki, T. Sakuma and T. Shibuya: Properties and Application Development of Shape Memory Alloy., 223-260 (CMC, Japan, 2001).

[2] T. Honma: Jour. Jpn. Soc. Mech. Eng., 87 517-522 (1984).

[3] K. Yamauchi: Jpn. Inst. Met., 7 495-449 (1993).

[4] M. Miyagi: Jpn. Inst. Met., 24 69-74 (1985).

[5] S. Miyazaki, T. Imai, Y. Igo, K. Otsuka, Met. Trans., 17A 115-120 (1986).

[6] T. Saburi: Metals and Technology., 59 11-18 (1989).

[7] S. Miyazaki and H. Sakamoto: Jpn. Inst. Met., 24 33-44 (1985).

[8] T. Todoroki and H. Tamura: J. Jpn. Inst. Met., 50 538-545 (1986).

[9] S. Miyazaki, Y. Ohmi, K. Otsuka, T. Suzuki, J. de Phys., C4-43 255-266 (1992).

[10] P. E. Thoma, D. R. Angst, K. D. Schachner, J. De Phys. Colloq., C8 577-562 (1995).

[11] D. N. Abujudom, P. E. Thoma, S. Fariabi, Met. Sci. Forum., 56-58 565-570 (1990).

[12] D. A. Miller, D. C. Lagoudas, Met. Sci. Eng., A308 161-175 (2001).

(Received December 28, 2009; Accepted April 20, 2010) 